\title{
38. Streptolysin S Formation by Streptococcal Ghosts
}

\author{
By Sadako Sugai and Fujio Egami \\ Department of Biophysics and Biochemistry, Faculty of Science, \\ University of Tokyo
}

(Comm. by Y. Shibata, M.J.A., March 12, 1960)

\begin{abstract}
Although it has been found that the production of streptolysin $\mathrm{S}$ by streptococci is greatly stimulated by the addition of oligonucleotides with high guanylic acid content, ${ }^{1)}$ the mechanism of the toxin formation remains to be elucidated. In order to clarify the mechanism, it seems necessary to obtain more simplified subcellular systems than intact cells. It has been reported previously that in the presence of suitable supporting media, streptococcal protoplasts can produce as much streptolysin $\mathrm{S}$ as intact cells. ${ }^{23)}$

Now it has been found that a subcellular fraction, so-called "ghosts", which is obtained by lysing protoplasts in hypotonic media, has an ability to produce streptolysin $\mathrm{S}$ under the conditions reported in this communication.
\end{abstract}

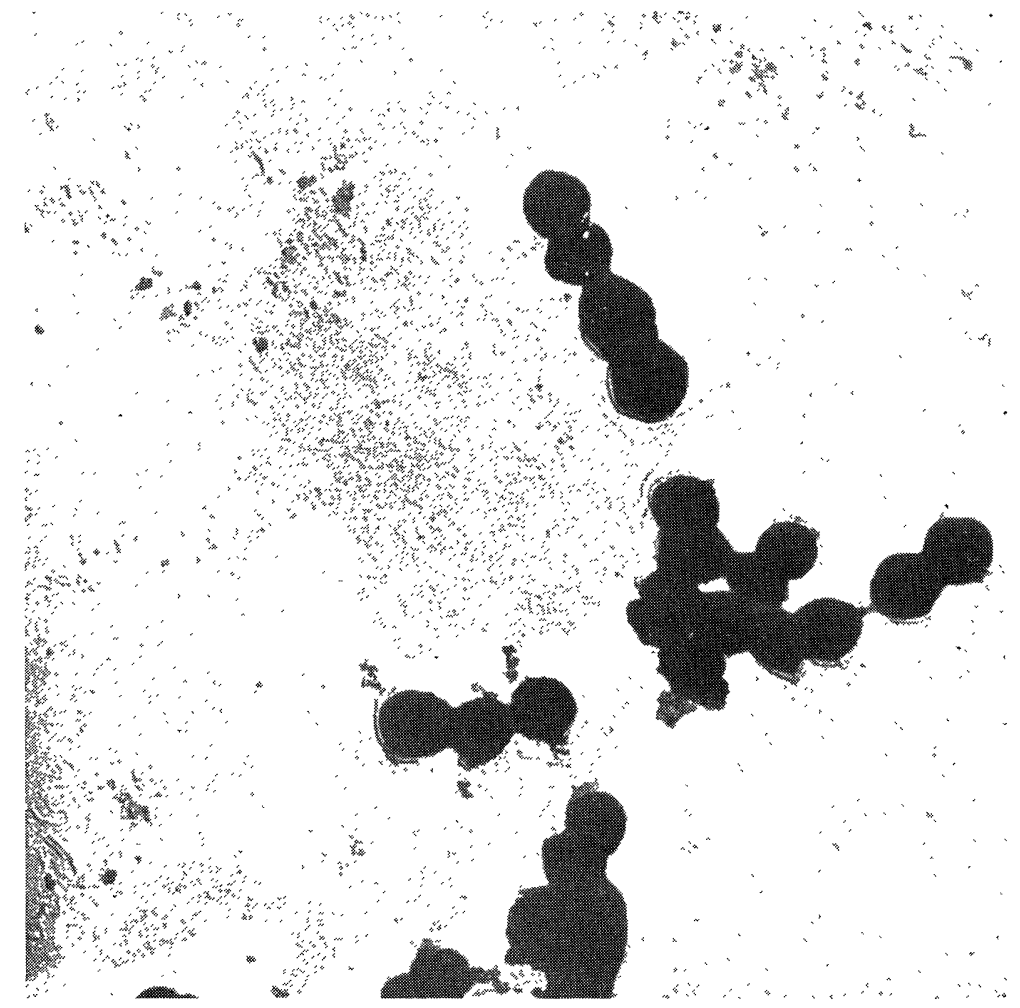

Fig. 1. (a) Electron micrograph of intact cells. $\times 10,000$ 


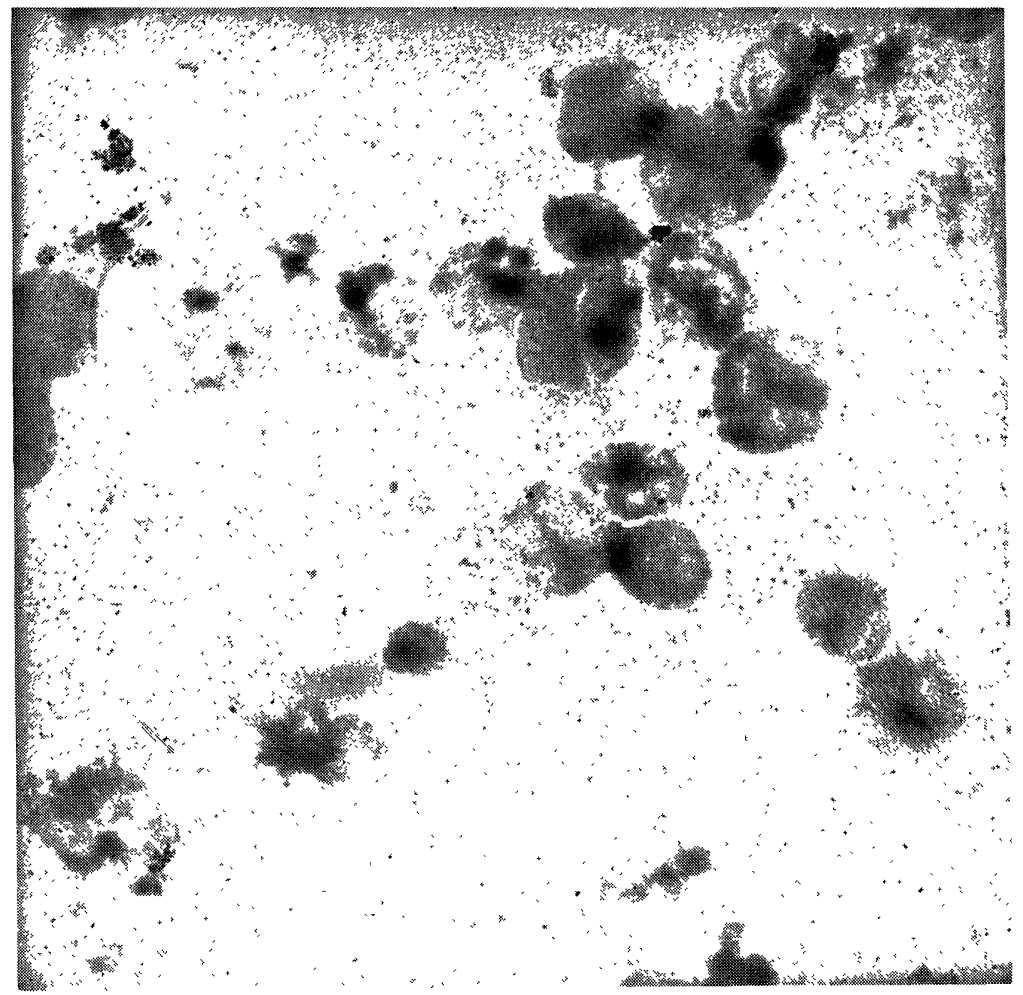

Fig. 1. (b) Electron micrograph of ghosts. $\times 10,000$

Protoplasts of Streptococcus pyogenes strain S 8 (group A hemolytic streptococcus) were prepared with the use of a lytic enzyme from a bacteriophage lysate of group $\mathrm{C}$ streptococcus in $0.5 \mathrm{M}$ succinate as a supporting medium. ${ }^{23)}$ To obtain ghosts, protoplasts were suspended in a medium of $\mathrm{pH} 7.0$ containing $0.2 \mathrm{M}$ succinate, $0.005 \mathrm{M}$ magnesium sulfate and $100 \mu \mathrm{gm}$ per $\mathrm{ml}$ of deoxyribonuclease, and incubated for 15 minutes at $37^{\circ} \mathrm{C}$. The highly viscous clump which appeared when protoplasts were suspended in the medium was dispersed by the action of deoxyribonuclease. After the incubation, the homogeneous suspension was centrifuged at 10,000 r.p.m. for 10 minutes in the cold. The pellet was washed with ice-cold phosphate buffer ( $\mathrm{pH} 7.0$ ) containing $0.005 \mathrm{M}$ magnesium sulfate and again separated by centrifugation. The pellet thus obtained consisted of ghosts and the electron micrograph of a typical preparation was given in Fig. 1.

Ghosts could produce streptolysin $\mathbf{S}$ when succinate in a concentration of $0.2 \mathrm{M}$ was present in the reaction medium. The optimum production was observed when $0.02 \mathrm{M}$ thioglycolate was added to the medium. Fig. 2 shows a typical time course of streptolysin $\mathrm{S}$ formation by the ghosts.

The necessity of succinate for streptolysin $\mathrm{S}$ formation by ghosts 


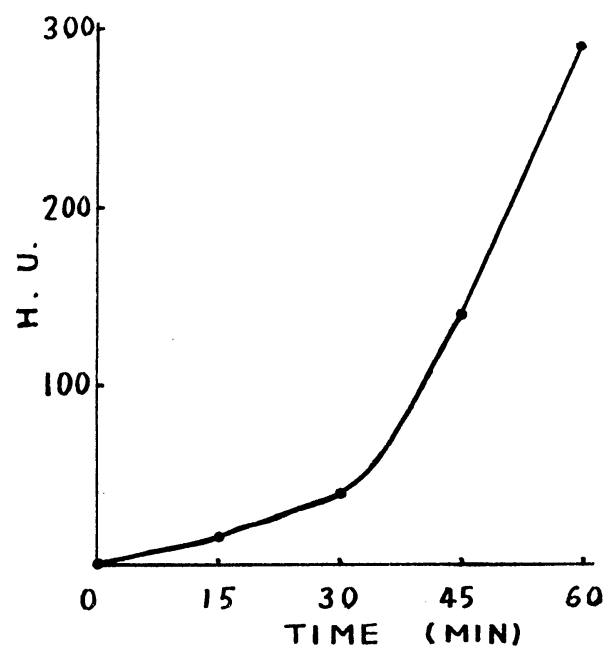

Fig. 2. Formation of streptolysin $\mathrm{S}$ by ghosts

The incubation mixture contained $2 \mu$ moles of magnesium sulfate, $30 \mu$ moles of potassium phosphate, $5 \mu$ moles of maltose, 0.2 mmole of sodium succinate, $20 \mu$ moles of thioglycolate, $100 \mu \mathrm{gms}$ of the active fraction ${ }^{1)}$ (the oligonucleotide fraction obtained by chromatography on "ECTEOLA" cellulose column of pancreatic ribonuclease core), and $2 \mathrm{mgm}$ dry weight of ghosts in a volume of $1 \mathrm{ml}, \mathrm{pH}$ being adjusted to 7.0. The reaction mixture was incubated at $37^{\circ} \mathrm{C}$ and at appropriate intervals aliquots were removed and the reaction was stopped by chilling. Ghosts were separated by centrifugation and the hemolytic activity in the supernatant was determined using a freshly prepared, 3 per cent rabbit erythrocytes suspension as described previously. ${ }^{23)}$ The hemolytic unit (H. U.) is the amount of hemolysin which will lyse half the erythrocytes contained in $1 \mathrm{ml}$ of phosphate buffered saline $(\mathrm{pH} 7.0)$ in $2 \mathrm{hr}$ at $37^{\circ} \mathrm{C}$.

(Table I) is in contrast with the case of intact cells, where succinate has a rather inhibitory effect. The function of succinate on streptolysin $\mathbf{S}$ formation by ghosts has not yet been elucidated, but it seems to have the effect of stabilizing the ghosts rather than to be an energy source, because malonate can replace succinate.

Table I. Requirements for streptolysin $\mathrm{S}$ formation by ghosts

\begin{tabular}{cc}
\hline Condition & Streptolysin S formed (H. U.) \\
\hline Complete system & 370 \\
Minus succinate & 0 \\
Minus thioglycolate & 34 \\
Minus maltose & 34 \\
Minus active fraction & 0 \\
\hline
\end{tabular}

Incubations were carried out at $37^{\circ} \mathrm{C}$ for 60 minutes. The composition of the complete system and the method of determination of hemolytic activity were described in Fig. 2. 
Table II. Effect of SH compounds

\begin{tabular}{lc}
\hline SH compound added & Streptolysin S formed (H. U.) \\
\hline None & 98 \\
Thioglycolate & 780 \\
Cysteine & 312 \\
Glutathione & 171 \\
\hline
\end{tabular}

The concentration of $\mathrm{SH}$ compounds was $0.02 \mathrm{M}$. For conditions of experiment and the expression of the results, see Fig. 2.

Thioglycolate increases also the production of streptolysin $\mathbf{S}$ by intact cells, but ghosts seem to be more sensitive to the reagent. Either cysteine or glutathione was effective as well as thioglycolate (Table II).

We wish to thank Dr. T. Nozima for the help for culture technique and to Mr. D. Okuno for taking electron micrographs. A part of the expense of this study was defrayed by a Grant from the Ministry of Education.

\section{References}

1) Tanaka, K.,: J. Biochem., 45, 109 (1958).

2) Maruyama, Y., Sugai, S., and Egami, F.,: Nature, 184, 832 (1959).

3) Maruyama, Y., Sugai, S., and Egami, F.,: Z. allgem. Mikrobiologie (1960) (in press). 